\section{(A) Check for updates}

Cite this: Analyst, 2021, 146, 4964

\title{
A study of diffraction-based chitosan leaky waveguide (LW) biosensors
}

\author{
Ruchi Gupta (D)*a and Nicholas J. Goddard ${ }^{b}$
}

The waveguide layer of diffraction-based leaky waveguides (LWs) must be made of materials that have low refractive index, are permeable to analytes, can be deposited by spin coating, and can be functionalised and crosslinked. These requirements are fulfilled by thin films of chitosan hydrogels. In this work, we studied the reproducibility of diffraction-based LWs with chitosan waveguides. The average refractive index sensitivity (RIS) and RI limit of detection (LOD) of the eight devices investigated herein were $125.5 \pm$ $3.8 \mathrm{deg} \mathrm{RIU}^{-1}$ and $1.9 \times 10^{-6} \pm 1.3 \times 10^{-6} \mathrm{RIU}$, respectively. While several challenges associated with the realisation of reproducible chitosan LWs have been addressed, reducing the variations in RI LOD requires improving the adhesion of chitosan films to glass substrates, minimising bubbles trapped in microfluidic channels, and using pumps with minimal pulsations. We showed that the buffer baseline of LWs with unmodified chitosan before and after introducing $750 \mu \mathrm{M}$ bovine serum albumin (BSA), which is equal to the physiological levels of serum albumin, was different by 3.6\%. Nevertheless, using biotin, anti-biotin antibody and BSA as exemplar recognition element, analyte and interferent, respectively, we demonstrated that diffraction-based chitosan LWs were suitable for monitoring analyte-RE binding in the presence of $750 \mu \mathrm{M}$ BSA.

Received 26th May 2021 Accepted 12 th July 2021

DOI: $10.1039 / \mathrm{d} 1 \mathrm{an} 00940 \mathrm{k}$ rsc.li/analyst
(FWHM) of each fringe at $\mathrm{LW}$ resonances is at least 10 times lower than FWHM of resonances of SPR, making it easier to determine small shifts in resonance angles observed at low analyte concentrations. ${ }^{12}$ Another benefit of LWs is that they are made of dielectric materials and hence can be hyphenated with electrokinetic methods ${ }^{13}$ for sample clean-up and analyte preconcentration. Finally, LWs can support transverse electric and transverse magnetic polarised optical modes ${ }^{14}$ and operate over the entire visible range of wavelengths,${ }^{15}$ providing information rich data.

Diffraction-based LWs require that the refractive index (RI) of the waveguide material is $<0.01$ higher than the sample solution, and porous hydrogels have a high potential to fulfil this requirement. Equally, analytes can diffuse in porous hydrogels and interact with a large fraction of the optical mode confined in the hydrogel waveguide. This contrasts with non-porous LWs where analytes only interact with the evanescent field of the optical mode confined in the waveguide. As a result, LWs made of porous hydrogels have been shown to offer 9 times greater refractive index sensitivity (RIS) that their non-porous equivalents. ${ }^{15}$ Additionally, the hydrogel precursor solutions should have appropriate viscosity so that thin films can be fabricated via spin coating, which results in much more uniform films than other methods such as casting. The application of LWs for biosensing applications demands that hydrogel films can be functionalised to attach REs and can be crosslinked for robust operations. Materials that can satisfy all

\footnotetext{
${ }^{a}$ School of Chemistry, University of Birmingham, Birmingham, B15 2TT, UK.

E-mail: r.gupta.3@bham.ac.uk

${ }^{b}$ Process Instruments (UK) Ltd, Turf Street, Burnley, BB11 3BP, UK.

E-mail:nick.goddard@processinstruments.net
} 
the above requirements are much harder to find in practise than it originally appears. For example, the authors have identified that RI of silica sol-gel, polystyrene, poly(2-hydroxyethyl methacrylate), poly(ethylene glycol) diacrylate films is high, spin coated films of agarose hydrogels are non-porous, viscosity of acrylamide/bisacrylamide solutions is too low to form polyacrylamide films by spin coating, and peptide and gelatine hydrogels are fragile.

Only chitosan hydrogels have so far shown promise towards fulfilling all the above requirements. ${ }^{6,16}$ Chitosan is a linear polysaccharide derived from partial or full deacetylation of chitin, which is an abundant and renewable natural resource. $^{17}$ Chitosan is non-toxic, biocompatible, biodegradable, ${ }^{18}$ antimicrobial, ${ }^{19,20}$ and film-forming, and hence has found widespread applications in food packaging, ${ }^{21}$ drug delivering, ${ }^{22,23}$ and sensing. ${ }^{24-27}$ Herein, we investigated the feasibility of making reproducible thin films of chitosan hydrogels, which served as the waveguide layer of diffractionbased LWs. Furthermore, we investigated the potential of unmodified chitosan films to resist non-specific adsorption (NSA) using bovine serum albumin (BSA) as a model protein at $750 \mu \mathrm{M}$, which is equal to the highest physiological level of albumin in serum. The findings of this work on the reproducibility of thin films of chitosan deposited on substrates and the ability of chitosan films to resist NSA are equally applicable to other types of sensors where thin films of hydrogels are used. Such sensors include SPR devices, fiber Bragg gratings, photonic crystals, Fabry Perot, and even electrochemical sensors.

\section{Experimental}

\section{Chemicals and materials}

Ethanol, $1 \mathrm{M}$ acetic acid, methanol, 25\% (v:v) glutaraldehyde (GA), (4-(2-hydroxyethyl)-1-piperazineethanesulfonic acid) (HEPES), $1 \mathrm{M}$ sodium hydroxide, anhydrous dimethyl sulfoxide, and bovine serum albumin (BSA) were bought from Sigma-Aldrich. Phosphate buffered saline (PBS, 10×) of pH 7.4 was bought from Generon. Decon 90, glycerol $\left(M_{\mathrm{w}}: 92\right)$ and chitosan ( $M_{\mathrm{w}}$ : $100000-300000$, 90\% deacetylated) were purchased from Fisher Scientific. Goat anti-biotin antibody (A150111A) was purchased from Cambridge Bioscience. Microscope glass slides and NHS-(PEG) 12 -biotin were bought from VWR. The bootlace ferrules (211-4252) that formed fluidic inlets/ outlets were purchased from RS Components and nitrile O-rings (01-08-01901) were bought from Ashton Seals.

\section{Fabrication of LWs and flowcell}

Glass slides were cut into $25.4 \times 25.4 \mathrm{~mm}^{2}$ squares, and cleaned in Decon 90, de-ionised water and ethanol for 30 minutes each followed by air plasma (PDC-002-CE expanded plasma cleaner, Harrick) for 15 min. Purified chitosan ${ }^{28}$ was dissolved in $0.1 \mathrm{M}$ acetic acid with magnetic stirring for $18 \mathrm{~h}$ to form $1 \%(\mathrm{w}: \mathrm{v})$ solution. $\sim 100 \mu \mathrm{l}$ of the chitosan solution was dispensed onto a glass square and spin coated at $900 \mathrm{rpm}$ with an acceleration of $100 \mathrm{rpm}$ for $30 \mathrm{~s}$. Each slide was then placed in an incubator at $75-80 \%$ humidity and $25{ }^{\circ} \mathrm{C}$ for $3 \mathrm{~min}$ and crosslinked by immersing in $0.03125 \%(\mathrm{v}: \mathrm{v})$ GA solution in $100 \mathrm{mM}$ HEPES buffer, $\mathrm{pH} 7.4$ for $10 \mathrm{~min}$. The slides were washed and stored in $100 \mathrm{mM}$ HEPES buffer, $\mathrm{pH}$ 7.4.

A flowcell with two microfluidic channels was made by computer numerical control (CNC) machining of $3 \mathrm{~mm}$ thick black poly(methyl methacrylate) forming $8.3 \mathrm{~mm}$ long, $1.9 \mathrm{~mm}$ wide, and $0.2 \mathrm{~mm}$ deep recessed cavities. Each cavity was surrounded by a groove $1 \mathrm{~mm}$ wide and $0.75 \mathrm{~mm}$ deep to mount an O-ring, and the two channels were separated by $2 \mathrm{~mm}$. Bootlace ferrules were glued in the through holes at the ends of each cavity to make inlets/outlets.

\section{Instrumentation}

The instrumentation used (see Fig. 1) has been described elsewhere, ${ }^{6}$ with the only major difference being the light source, which was a $475 \mathrm{~nm}$ LED (Lumileds LUXEON Z LXZ1-PB01, RS Components). The output of the LED was coupled to a $200 \mu \mathrm{m}$ core multimode optical fibre, which was collimated using an achromatic doublet (63DQ25, Comar Optics Ltd) and then converted to a wedge beam using a cylindrical lens (63YD25, Comar Optics Ltd). The light beam was coupled to the LW device using an NBK7 equilateral prism with square faces of $30 \times 30 \mathrm{~mm}$. A thin layer of RI matching oil (Cargille $^{\mathrm{TM}}$ Immersion Oil Type A, Fisher Scientific) was placed between the LW and prism. The light reflected from each point on the LW was recorded on a CMOS camera (Mercury Daheng MER-2000-19U3M, GeT Cameras BV) to obtain 2D-reflectivity curves. The camera had 5496 by 3672 pixels with each pixel being $2.4 \times 2.4 \mu^{2}$. The instrument and camera control software were used to control the angular positions of the LED and camera, driving current of the LED, and real-time processing of $2 \mathrm{D}$ reflectivity curves to display a chart of shift in resonance angles of LWs versus time. The solutions were pumped using a MINIPULS 3 peristaltic pump (Gilson) at a volumetric flow rate of $3 \mu \mathrm{L} \mathrm{s}^{-1}$. The RI of solutions were measured using RFM900-T refractometer (Bellingham and Stanley) with an

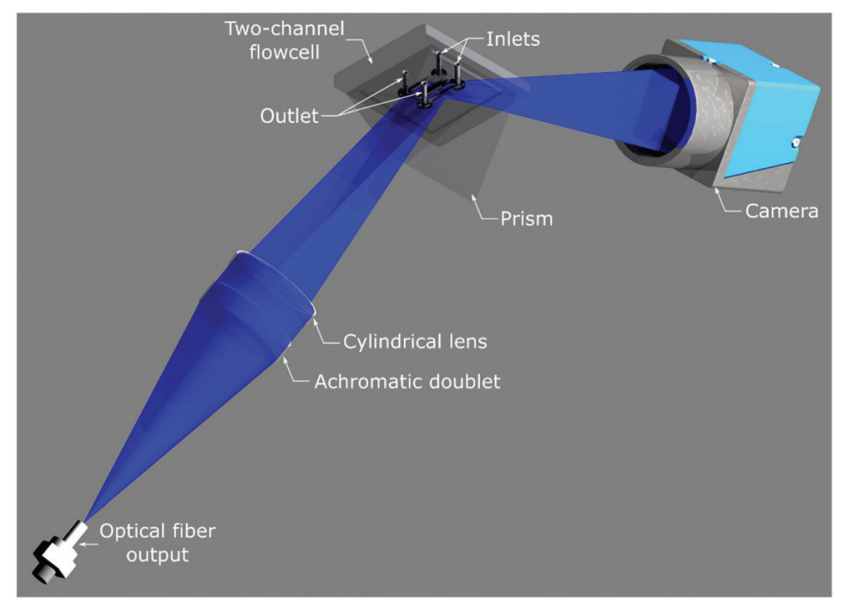

Fig. 1 Instrumentation with a two-channel flowcell on top of the LW and prism assembly. 
accuracy of $\pm 1 \times 10^{-5}$. All solutions were prepared in $10 \mathrm{mM}$ PBS, pH 7.4.

\section{Results and discussion}

\section{LW device 5}

$2 \mathrm{D}$ - and 1D-reflectivity curves of one of the LWs are shown in Fig. 2 where device 5 was selected to show the worst-case. The positions of TIR and resonance (LW1 and LW0) angles are also marked in Fig. 2. As discussed previously, ${ }^{7}$ exponentially decaying interference fringes are observed in reflectivity curves at resonance angles because of Fresnel diffraction. The number of fringes observed depends on the bandwidth of the light source, the RI of the waveguide and any optical losses in the LW. ${ }^{7}$ In the case of chitosan LWs, as shown in Fig. 2, only one dip-peak pair of exponentially decaying interference fringes was observed at each resonance angle. Additionally, two sets of dip-peak pairs were observed in Fig. 2, which in turn suggests that the chitosan film was sufficiently thick to support two optical modes. The positions of dips were determined using a centre of gravity algorithm and were taken as a measure of the position of the resonance angles. Only the position of LW0 was studied for the remainder of this work.

The mean positions of LW0 in channels 1 and 2 were $63.827 \pm 0.005 \mathrm{deg}$ and $63.820 \pm 0.017 \mathrm{deg}$ (both: $n=4$, i.e., areas in each channel), respectively. As the variation in the position of LW0 between the two channels was low, the thickness of the chitosan film must be uniform. To verify this, the thickness and RI of the chitosan film were estimated using an in-house transfer matrix-modelling package. ${ }^{7}$ The estimated average thicknesses of chitosan for channels 1 and 2 were 2.689 and $2.687 \mu \mathrm{m}$, respectively. Thus, the difference in the thickness of chitosan in the two channels was $0.05 \%$. Furthermore, the average RI of chitosan for channels 1 and 2 were $1.347-0.0002 \mathrm{i}$ and $1.347-0.00015 \mathrm{i}$, respectively.
The RIS of device 5 was determined by measuring shifts in LW0 for glycerol solutions of different concentrations and hence RI, which were measured independently using a refractometer. As shown in Fig. 3(a), shifts in LW0 increased as higher concentrations/RI of glycerol solutions were introduced on the LW. The relationships between shifts in LW0 $\left(\Delta \theta_{\mathrm{R}}\right)$ and $n_{\text {gly }}$ for both channels were linear, and are given by eqn (1) and (2) with $r^{2}$ of 0.9927 and 0.9922 , respectively.

Channel 1:

$$
\Delta \theta_{\mathrm{R}}=-172.9+129.5 \times n_{\mathrm{gly}}
$$

Channel 2:

$$
\Delta \theta_{\mathrm{R}}=-172.1+128.9 \times n_{\mathrm{gly}}
$$

The slope of the regression line between $\Delta \theta_{\mathrm{R}}$ and $n_{\text {gly }}$ provides the RIS of LWs. Thus, the RIS of the device corresponding to channels 1 and 2 were $129.5 \pm 5.6 \mathrm{deg} \mathrm{RIU}^{-1}$ and $128.9 \pm 5.7 \mathrm{deg}$ $\mathrm{RIU}^{-1}$ (both: $n=4$, i.e., total number of areas in each channel), respectively where the errors represent area-to-area variability within each channel. The identical RIS in the two channels was a result of the uniform thickness and RI of chitosan. The identical RIS of the two channels is beneficial for eliminating commonmode effects (e.g., changes in temperature and sample composition) by performing differential measurements between the two channels. Based on the similarity in the RIS of the two channels, common-mode effects can be reduced by $99.7 \%$.

Based on Fig. 2, the average FWHMs of the LW0 mode for channels 1 and 2 were 0.077 and $0.107 \mathrm{deg}$, respectively. The narrower the FWHM, the easier it is to monitor small shifts in resonance angles, which are observed at low analyte concentrations. ${ }^{12}$ For device 5, the figure of Merit (FoM = RIS/FWHM) was 1635 and $1179 \mathrm{RIU}^{-1}$ for channels 1 and 2, respectively. To determine the RI LOD, the standard deviation of shifts in LW0 of each channel for all concentrations of glycerol was calculated. The exemplar data used to calculate the standard devi- (a)

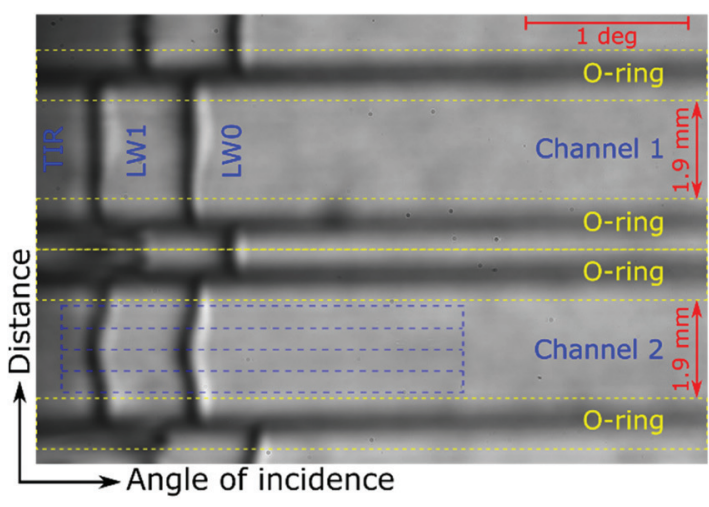

(b)
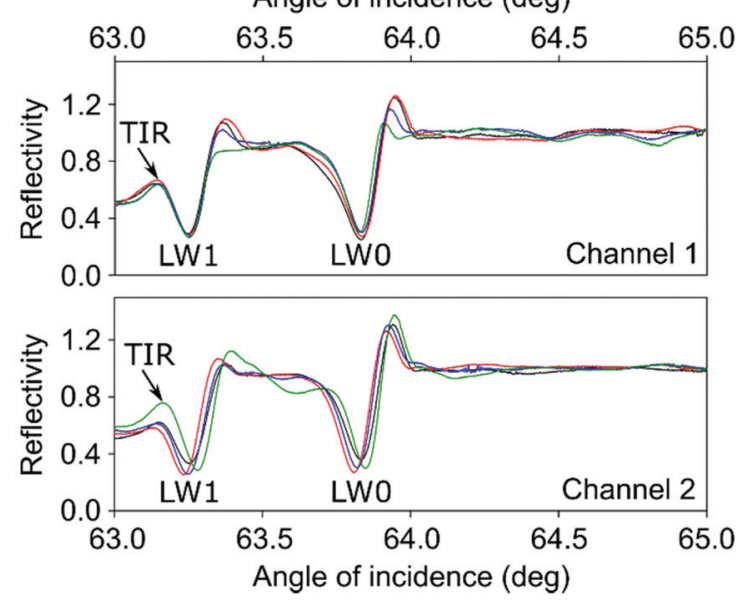

Fig. 2 (a) 2D- (where dotted blue lines show the 4 areas in channel 2) and (b) corresponding 1D-reflectivity (different colour traces correspond to different areas in each channel) profiles of device 5 . 

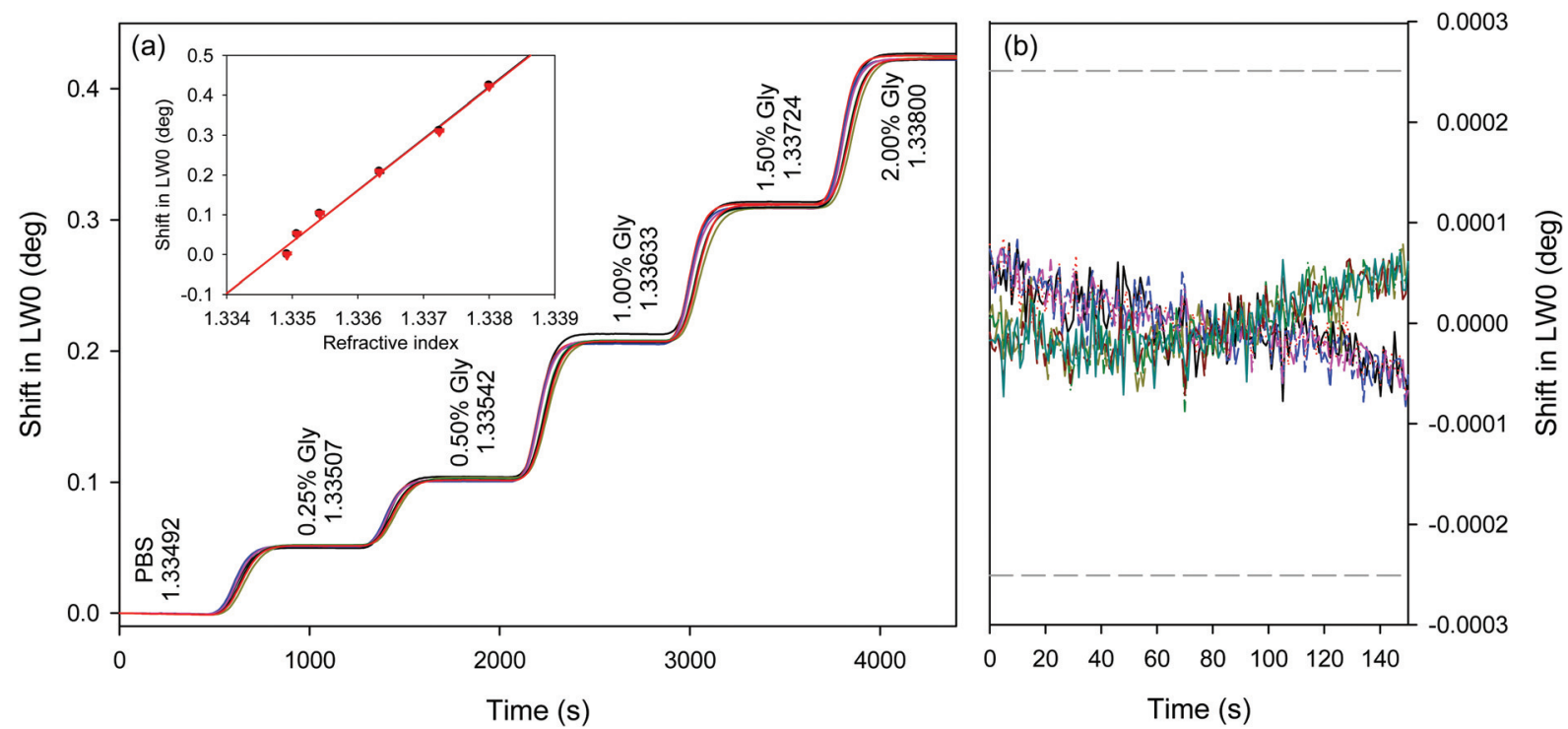

Fig. 3 (a) A sensorgram of device 5 for glycerol solutions (different colour traces correspond to different areas in each channel) where the inset is a plot of shift in LWO versus RI of glycerol solutions, and (b) exemplar data used to calculate the standard deviation of shifts in LWO where dashed lines represent shift in LWO (deg) corresponding to $\pm 2 \times 10^{-6}$ RIU.

ation of shifts in LW0 is provided in Fig. 3(b). The standard deviations of shifts in LW0 of both channels were divided by RIS of the device to obtain the RI LOD. For device 5 , the RI LOD for channels 1 and 2 were $2.8 \times 10^{-6} \pm 1.6 \times 10^{-6}$ RIU and $3.4 \times 10^{-6} \pm 3.7 \times 10^{-6} \mathrm{RIU}$ (both: $n=24$, i.e., product of six glycerol concentrations and four areas in each channel), respectively. The standard deviation of RI LOD is a measure of the noise in the sensorgrams. The primary source of noise was the pulsation of the peristaltic pump and increased when air bubbles were trapped in the microfluidic channels despite these bubbles being out of the region where the light beam was falling. This noise can therefore be reduced by computational methods such as Fourier filtering or by using syringe pumps or even gravity feed. ${ }^{29}$

\section{Area-to-area and device-to-device reproducibility}

Glycerol runs were performed for eight LWs to determine their RIS, and the results are summarised in Table 1. The area-to-

Table 1 Summary of RIS and RI LOD of the eight LW devices studied in this work (where error bars in in RIS represent area-to-area variations and error bars in RI LOD represent noise)

\begin{tabular}{llllll}
\hline & \multicolumn{2}{l}{$\begin{array}{l}\text { RIS }\left(\mathrm{deg} \mathrm{RIU}^{-1}\right) \\
(n=4)\end{array}$} & & \multicolumn{2}{l}{$\begin{array}{l}\text { RI LOD }\left(\times 10^{-6} \mathrm{RIU}\right) \\
(n=12)\end{array}$} \\
\cline { 2 - 3 } \cline { 5 - 6 } Devices & Channel 1 & Channel 2 & & Channel 1 & Channel 2 \\
\hline Device 1 & $120.8 \pm 2.6$ & $118.2 \pm 2.5$ & & $1.4 \pm 0.6$ & $1.5 \pm 1.0$ \\
Device 2 & $126.6 \pm 1.8$ & $126.2 \pm 1.9$ & & $1.8 \pm 0.5$ & $2.9 \pm 0.2$ \\
Device 3 & $125.4 \pm 4.6$ & $125.4 \pm 3.8$ & & $2.0 \pm 0.8$ & $2.2 \pm 0.7$ \\
Device 4 & $125.4 \pm 4.6$ & $124.6 \pm 4.4$ & & $2.3 \pm 1.1$ & $2.4 \pm 1.5$ \\
Device 5 & $129.5 \pm 5.6$ & $128.9 \pm 5.7$ & & $2.8 \pm 1.6$ & $3.4 \pm 3.7$ \\
Device 6 & $125.0 \pm 5.7$ & $124.9 \pm 5.7$ & & $0.9 \pm 0.2$ & $0.9 \pm 0.1$ \\
Device 7 & $128.3 \pm 2.2$ & $128.3 \pm 2.2$ & & $1.4 \pm 0.5$ & $1.1 \pm 0.6$ \\
Device 8 & $125.2 \pm 2.0$ & $124.0 \pm 2.0$ & & $1.5 \pm 1.5$ & $1.5 \pm 0.9$
\end{tabular}

area variability in each channel was between $1.4 \%$ and $4.6 \%$. A statistical $t$-test suggested that the mean RIS of channels 1 and 2 of each LW were not significantly different at 95\% confidence level. The mean RIS of the eight devices was $125.5 \pm$ $3.8 \mathrm{deg} \mathrm{RIU}^{-1}$. The mean RIS of devices 2 to 8 were not significantly different at the $95 \%$ confidence level, but device 1 was significantly different from devices $2,35,7$ and 8 . One possible reason for this difference is that device 1 was conditioned with flowing PBS buffer for $\sim 1 \mathrm{~h}$ compared to other devices, which were conditioned for $\sim 5 \mathrm{~h}$. Conditioning was required because the devices were stored in HEPES buffer but PBS was used for the experimental work to better mimic physiological conditions. After device 1 , the conditioning time was increased to reduce the baseline drift which was continuing after one hour.

The typical area-to-area variability in the RI LOD was between $14.3 \%$ and $64.2 \%$. The area-to-area variability in the RI LOD of channel 2 of device 5 and channel 1 of device 8 was $>100 \%$. As shown in Fig. 2, the position of LW0 across the width of channel 2 of device 5 was less uniform than channel 1 , suggesting that defects must have been introduced during the fabrication of the chitosan film. Similarly, we observed that the chitosan film in channel 1 of device 8 washed away after $\sim 8 \mathrm{~h}$, which was in contrast to other devices where chitosan films remained intact for days. As the standard deviation of RI LOD was device dependent, in addition to the pulsation of the peristaltic pump, it must also be a function of the quality of the chitosan films deposited on glass substrates. We hypothesise that if regions of chitosan films have detached from glass substrates, noise may be amplified because the gap between chitosan and glass substrates may oscillate with the pulsation of the peristaltic pump. The adhesion between chitosan and glass substrates may be improved by attaching them 
covalently. Overall, the average RI LOD of all eight devices was $1.9 \times 10^{-6} \pm 1.3 \times 10^{-6} \mathrm{RIU}$.

\section{NSA of BSA to chitosan}

BSA was selected because it is a widely used protein to investigate NSA. ${ }^{30,31}$ Furthermore, the highest concentration of BSA solution used was equivalent to the concentration of albumin in human serum. ${ }^{32,33}$ To determine whether BSA showed significant NSA to chitosan, BSA was injected in the sensor channel and buffer was flowed in the reference channel and shifts in LW0 of sensor channel was recorded with respect to the reference channel. The observed differential shifts in LW0 $\left(\Delta \theta_{\mathrm{R}, \mathrm{obs}}\right)$ at equilibrium are summarised in Table 2 . Table 2 also lists expected shifts in LW0 $\left(\Delta \theta_{\mathrm{R}, \exp }\right)$, which were determined using eqn (1) and assumes that chitosan films are completely porous to BSA. In eqn (3), RIS $_{\text {gly }}$ is refractive index sensitivity of LWs determined using glycerol solutions, $n_{\mathrm{c}}$ and $n_{0}$ are RI of BSA solution of selected concentration and buffer, respectively.

$$
\Delta \theta_{\mathrm{R}, \exp }=\operatorname{RIS}_{\text {gly }}\left(n_{\mathrm{c}}-n_{0}\right)
$$

The observed and expected shifts in LW0 were expected to be comparable if chitosan films are completely porous to BSA, and BSA does not NSA to chitosan. However, Table 2 shows that the observed shift in LW0 was significantly lower than expected at low BSA concentrations. This in turn suggests that chitosan films were not fully permeable to BSA. Equally, Table 2 shows that as the BSA concentration was increased, the difference between the observed and expected shifts in LW0 decreased until at $750 \mu \mathrm{M}$ BSA the shift became slightly larger than expected. It is unlikely that the porosity of chitosan changes so markedly to different BSA concentrations, so the most likely explanation is that at high concentrations BSA binds to chitosan and fills all the pores of the LW and then starts adsorbing to the surface of the chitosan LW. BSA is known to show multilayer adsorption, ${ }^{34}$ and has a RI of about 1.507 in multilayers. ${ }^{35}$ This means that it has a higher RI than the chitosan, and thus is no longer in the evanescent field of the LW but forms part of the waveguide. This would account for the larger than expected resonance angle shifts at high BSA concentrations. Modelling indicates that multilayer adsorption in the pores and on the surface of the waveguide can account for the non-linearity of the response to BSA compared to nonadsorbing glycerol. Based on the estimated RI of hydrated BSA, Fig. 4 shows the number of layers of BSA as a function of the protein concentration. From this, it can be estimated that the coverage of BSA will saturate at $\sim 25$ layers.

The difference in shifts in LW0 with PBS before introducing BSA and after washing out the protein (i.e., the last column of Table 2) increased as the concentration of BSA solution changed from 2.5 to $750 \mu \mathrm{M}$. Based on the values in the last column of Table 2, at BSA concentrations of $75 \mu \mathrm{M}$ and above, washing with buffer could not remove all the BSA in $800 \mathrm{~s}$. This indicates that at high concentrations of BSA, there is some largely irreversible NSA of BSA to chitosan films. At $\mathrm{pH}$ 7.4, the NSA of BSA to chitosan may be because of electrostatic attraction as BSA will be negatively charged (pI: 4.5-5.0 ${ }^{36}$ ) and chitosan will be positively charged $\left(\mathrm{p} K_{\mathrm{a}}: 6.5-6.6^{37}\right)$.

\section{RE-analyte binding}

The sensor region of a LW was functionalised with biotin by reacting amine groups of chitosan with $N$-hydroxysuccinimide (NHS) group of NHS-(PEG) 12 -biotin. For this purpose, 50 $\mu \mathrm{g} \mathrm{ml} \mathrm{m}^{-1}$ NHS-(PEG) ${ }_{12}$-biotin solution was pumped in the sensor channel for $30 \mathrm{~min}$. During this time, PBS was pumped

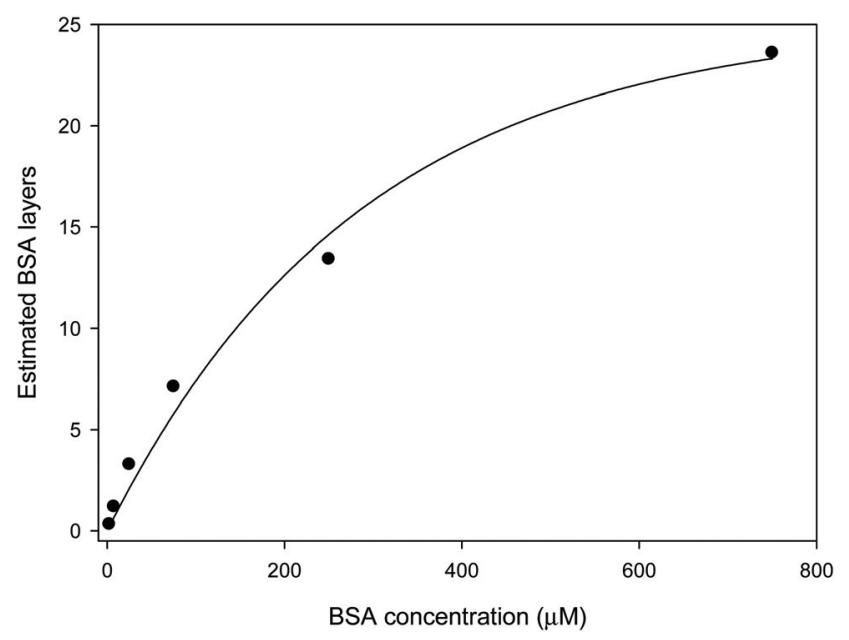

Fig. 4 Estimated number of BSA layers deposited on LW as a function of the protein concentration.

Table 2 Summary of shifts in LWO in the presence of BSA solutions and PBS (where $n=$ total number of areas per channel and error bars represent area-to-area variations of LW device 6)

\begin{tabular}{|c|c|c|c|c|}
\hline \multirow[b]{2}{*}{ BSA concentration $(\mu \mathrm{M})$} & \multirow[b]{2}{*}{ RI } & \multicolumn{2}{|c|}{ Shifts in LW0 $\left(\times 10^{-3} \mathrm{deg}\right)$ for BSA $(n=4)$} & \multirow{2}{*}{$\begin{array}{l}\text { Difference between shifts in LW0 } \\
\left(\times 10^{-3} \mathrm{deg}\right) \text { for PBS before and after BSA }(n=4)\end{array}$} \\
\hline & & Observed $\left(\Delta \theta_{\mathrm{R}, \mathrm{obs}}\right)$ & Expected $\left(\Delta \theta_{\mathrm{R}, \exp }\right)$ & \\
\hline 0 (Buffer) & 1.33473 & Not Applicable & & Not Applicable \\
\hline 2.5 & 1.33480 & $2.2 \pm 0.1$ & 8.8 & $-3.2 \pm 0.2$ \\
\hline 7.5 & 1.33492 & $8.4 \pm 0.1$ & 23.7 & $-2.8 \pm 0.1$ \\
\hline 25 & 1.33504 & $26.7 \pm 0.3$ & 38.7 & $-2.3 \pm 0.1$ \\
\hline 75 & 1.33567 & $82.0 \pm 0.5$ & 117.4 & $0.3 \pm 0.4$ \\
\hline 250 & 1.33759 & $306.3 \pm 1.1$ & 357.3 & $9.9 \pm 0.4$ \\
\hline 750 & 1.34390 & $1175.1 \pm 0.5$ & 1145.6 & $41.1 \pm 0.3$ \\
\hline
\end{tabular}



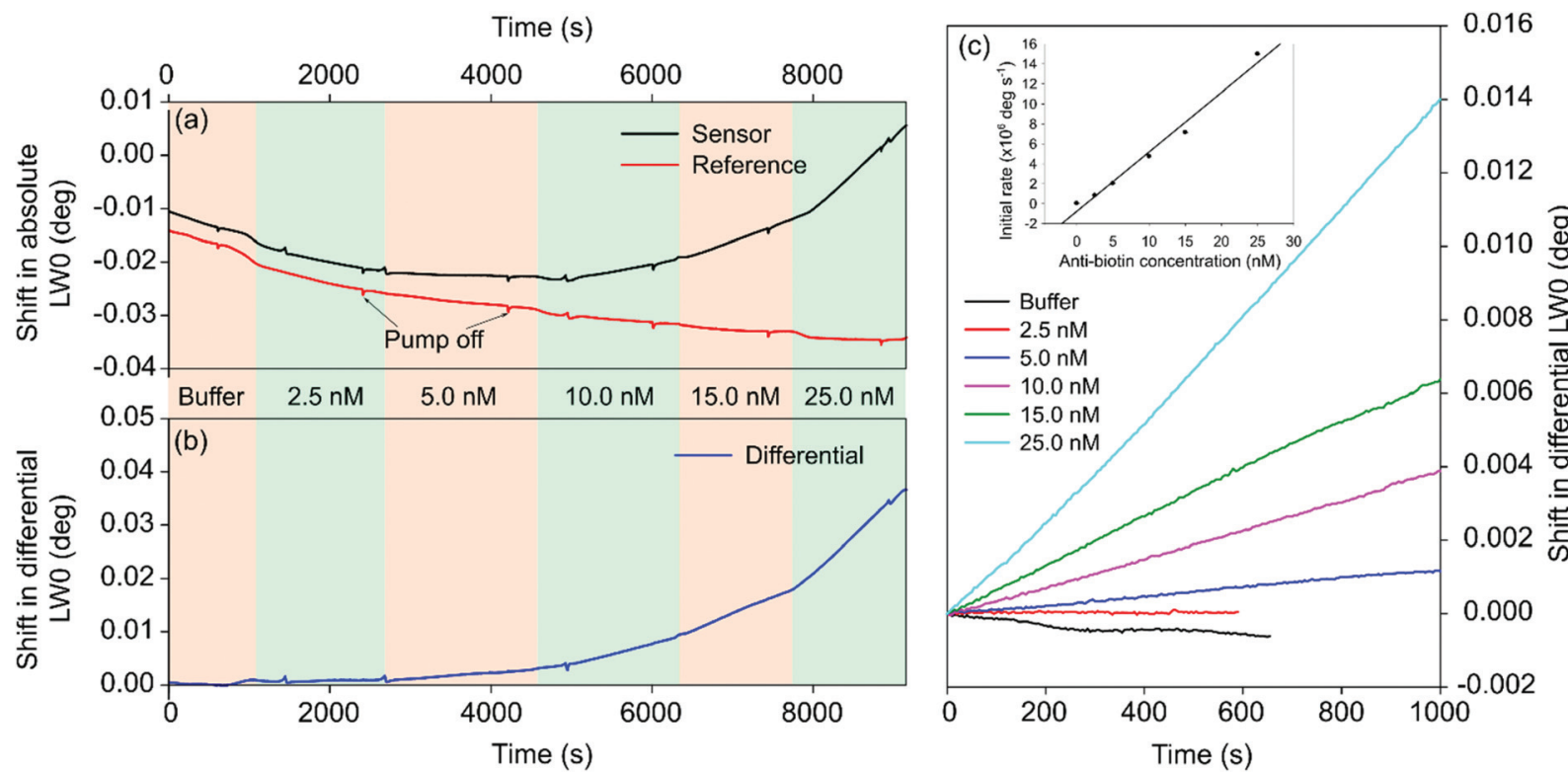

Fig. 5 Shifts in (a) absolute and differential resonance angle in (b) real-time, and (c) after being made to pass through origin (inset shows a plot of initial rate of binding versus concentrations of anti-biotin antibody solutions and errors bars are too small to be visible, device 1).

in the reference channel, leaving the reference region unmodified. 0.0, 2.5, 5.0, 10.0, 15.0 and $25.0 \mathrm{nM}$ anti-biotin antibody solutions prepared in PBS were introduced sequentially and shifts in resonance angles of both channels were monitored. Fig. 5(a) shows that the resonance angle of the reference decreases with time, which is attributed to an increase in the ambient temperature. Consequently, the binding of anti-biotin antibody at low concentrations was less apparent in the absolute shift in resonance angle of the sensor (black trace in Fig. 5(a)). Glitches in traces in Fig. 5(a) were also observed when the peristaltic pump was turned off briefly to switch from one solution to another. As shown in Fig. 5(b), the downward drift and glitches were significantly reduced in the shifts in differential resonance angle, which was obtained by subtracting shifts in absolute resonance angles of the sensor and reference. This in turn suggests that differential measurements between closely spaced sensor and reference channels were effective in removing the effect of changes in temperature and perturbations in flow rate.

A corresponding plot of shifts in differential LW0 is provided in Fig. 5(c) where the time at which anti-biotin antibody solution of each concentration was injected was taken as zero time point. Additionally, the differential LW0 at each zero time point was subtracted from all subsequent values. The inset in Fig. 5(c) clearly shows that the rate of increase in the shift in differential resonance angle increased linearly with antibody concentration from buffer to $25.0 \mathrm{nM}$. Since we are in the laminar flow regime (Reynolds number $\sim 1$ ), the Nernst diffusion layer treatment can be applied. Eddowes ${ }^{38}$ showed that in this regime, at low surface coverage, the initial rate is proportional to the bulk antibody concentration.

The relationship between the rate of increase in the shift in differential resonance angle $\left(\mathrm{d} \Delta \theta_{\mathrm{R}} / \mathrm{d} t\right)$ and concentration of anti-biotin antibody solution $\left(c_{\mathrm{Ab}}\right)$ (see the inset in Fig. 5(c)) is given by $\mathrm{d} \Delta \theta_{\mathrm{R}} / \mathrm{d} t=-7.7 \times 10^{-7}+6.0 \times 10^{-7} \times c_{\mathrm{Ab}}\left(r^{2}=0.9831\right)$. Thus, the initial rate of binding per unit concentration of antibiotin antibody was $6.0 \times 10^{-7} \mathrm{deg} \mathrm{s}^{-1} \mathrm{nM}^{-1}$. Different concentrations of anti-biotin antibody solutions prepared in PBS containing $3.76 \mu \mathrm{M}$ BSA were then sequentially introduced in the sensor and reference channels of a fresh LW. In this case, the relationship between $\mathrm{d} \Delta \theta_{\mathrm{R}} / \mathrm{d} t$ and $c_{\mathrm{Ab}}$ is given by $\mathrm{d} \Delta \theta_{\mathrm{R}} / \mathrm{d} t=$ $-21.4 \times 10^{-7}+7.2 \times 10^{-7} \times c_{\mathrm{Ab}}\left(r^{2}=0.9677\right)$. Thus, the initial rate of binding per unit concentration of anti-biotin antibody was $7.2 \times 10^{-7} \mathrm{deg} \mathrm{s}^{-1} \mathrm{nM}^{-1}$, which was $\sim 20 \%$ higher than the case when the anti-biotin antibody solutions were prepared in PBS. The difference between the values obtained without and with BSA suggest that BSA may be non-specifically adsorbed more to the sensor than the reference regions, either to biotin or bound antibodies, and will be studied in future work.

Finally, $25 \mathrm{nM}$ anti-biotin antibody solution was prepared in PBS containing $750 \mu \mathrm{M}$ BSA, and was pumped in both the sensor and reference channels of a fresh LW. Fig. 6(a) shows the shifts in LW0 of both the sensor and reference channels increased with time. While the shift in the LW0 of the reference channel is likely to be because of NSA of BSA to chitosan, the shifts in the LW0 of the reference channel is the sum of NSA of BSA and binding of the antibody to biotin molecules immobilised in the chitosan waveguide. The contribution of the shift in LW0 because of NSA of BSA was largely eliminated by taking differential measurements between the sensor and reference channels (Fig. 6(b)). In this case, the relationship between the shift in differential resonance angle $\left(\mathrm{d} \Delta \theta_{\mathrm{R}}\right)$ and time $(t)$ is given by $\Delta \theta_{\mathrm{R}}=-28.4 \times 10^{-5}+1.9 \times 10^{-5} \times t\left(r^{2}=\right.$ 0.9951). Thus, the initial rate of binding per unit concentration of antibody was $7.4 \times 10^{-7} \mathrm{deg} \mathrm{s}^{-1} \mathrm{nM}^{-1}$, which is comparable to the value when the anti-biotin antibody solutions were pre- 

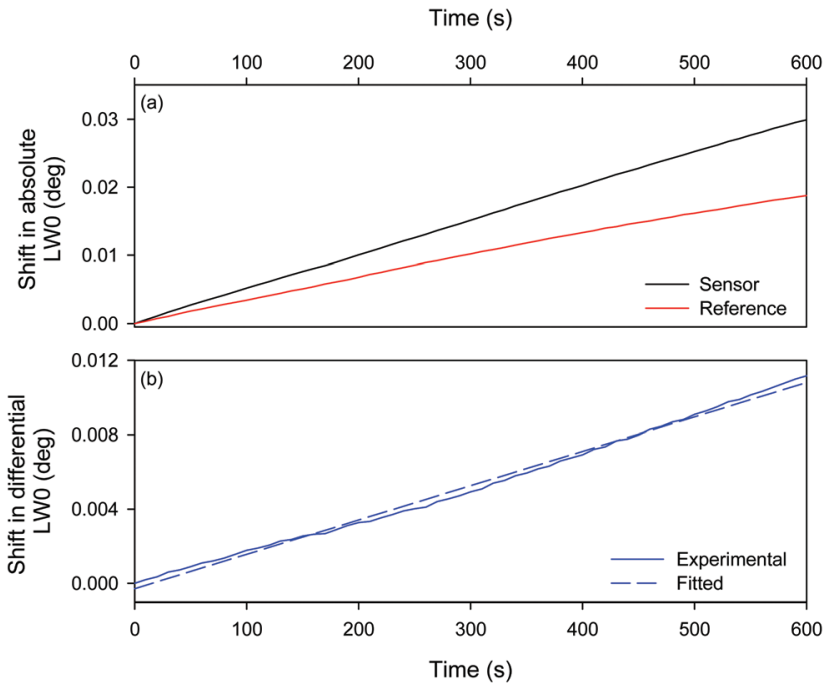

Fig. 6 (a) Absolute and (b) differential shifts in LWO on irrigating the sensor and reference channels with $25 \mathrm{nM}$ anti-biotin antibody solution containing $750 \mu \mathrm{M}$ BSA (device 7).

pared in $3.76 \mu \mathrm{M}$ BSA. Because the non-specific (weak) binding of BSA happens rapidly at high concentrations, the much slower binding of the low concentration analyte can be distinguished by rate measurements after the step change caused by BSA.

\section{Conclusions}

Diffraction-based leaky waveguides (LWs) are the simplest possible biosensors comprised of a few microns thick hydrogel film on a glass substrate. In this work, we studied eight diffraction-based LWs made of chitosan and showed that the area-toarea and device-to-device reproducibility in the refractive index sensitivity (RIS) of all except one device was high. Our study suggested that the time for which LWs were conditioned in buffer before use played an important role in determining their RIS. In contrast, the area-to-area and device-to-device reproducibility of the refractive index (RI) limit of detection (LOD) is currently low and must be improved. Possible ways for improving the reproducibility of RI LOD include covalent attachment of chitosan films to glass substrates, Fourier filtering of sensorgrams to remove pump noise, and replacing the peristaltic pump with a syringe pump or gravity feed. The studies on non-specific adsorption (NSA) of bovine serum albumin (BSA) to chitosan LWs suggested that the protein solutions of up to $25 \mu \mathrm{M}$ interacted with chitosan reversibly and the BSA could be largely washed out by flowing $10 \mathrm{mM}$ PBS over the chitosan films. In comparison, BSA solutions between 75 and $750 \mu \mathrm{M}$ showed multilayer adsorption to chitosan. The initial rate of antibody binding has been shown to be linear up to $25 \mathrm{nM}$ in the presence and absence of BSA as a non-specifically adsorbing interferent. Antibody binding could be distinguished from NSA of BSA even at $750 \mu \mathrm{M}$, a $30000-$ fold excess over the maximum antibody concentration, because the step change caused by NSA of BSA happened quickly and was followed by the much slower binding of the analyte. This indicates that chitosan LW sensors could potentially detect analytes in undiluted serum.

\section{Author contributions}

Both authors contribute equally to this work.

\section{Conflicts of interest}

The authors declare no conflict of interest.

\section{Acknowledgements}

The authors acknowledge the funding support from the Engineering and Physical Sciences Research Council (EPSRC, Grant EP/N02074X/2). The authors also thank Ms Hazel J. Dixon for fabricating chitosan films.

\section{References}

1 S. Lofas and B. Johnsson, J. Chem. Soc. Chem. Commun., 1990, 1526, DOI: 10.1039/c39900001526.

2 B. Johnsson, S. Lofas and G. Lindquist, Anal. Biochem., 1991, 198, 268.

3 U. Jönsson, L. Fägerstam, B. Ivarsson, B. Johnsson, R. Karlsson, K. Lundh, S. Löfås, B. Persson, H. Roos, I. Rönnberg, S. Sjolander, E. Stenberg, R. Stahlberg, C. Urbaniczky, H. Ostlin and M. Malmqvist, BioTechniques, 1991, 11, 620.

4 S. Lofas, M. Malmqvist, I. Ronnberg, E. Stenberg, B. Liedberg and I. Lundstrom, Sens. Actuators, B, 1991, 5, 79.

5 B. Liedberg, I. Lundstrom and E. Stenberg, Sens. Actuators, $B, 1993,11,63$.

6 A. K. Pal, N. J. Goddard, H. J. Dixon and R. Gupta, Biosensors, 2020, 10, 134.

7 N. J. Goddard and R. Gupta, Sens. Actuators, B, 2020, 309, 127776.

8 N. A. Alamrani, G. M. Greenway, N. Pamme, N. J. Goddard and R. Gupta, Analyst, 2019, 144, 6048.

9 R. Gupta and N. J. Goddard, 17th International Conference on Miniaturized Systems for Chemistry and Life Sciences (MicroTAS 2013), Freiburg, Germany, 2013, vol. 3, p. 1490.

10 R. Gupta and N. J. Goddard, Analyst, 2013, 138, 1803.

11 V. N. Konopsky and E. V. Alieva, Anal. Chem., 2007, 79, 4729.

12 R. Gupta and N. J. Goddard, Sens. Actuators, B, 2020, 322, 128628.

13 N. J. Goddard and R. Gupta, Sens. Actuators, B, 2019, 301, 127063.

14 Z. Salamon, M. I. Brown and G. Tollin, Trends Biochem. Sci., 1999, 24, 213. 
15 R. Gupta, N. A. Alamrani, G. M. Greenway, N. Pamme and N. J. Goddard, Anal. Chem., 2019, 91, 7366.

16 N. J. Goddard and R. Gupta, IEEE Trans. Instrum. Meas., 2021, 70, 7502612 .

17 D. Elieh-Ali-Komi and M. R. Hamblin, Int. J. Adv. Res., 2016, 4, 411.

18 S. K. Shukla, A. K. Mishra, O. A. Arotiba and B. B. Mamba, Int. J. Biol. Macromol., 2013, 59, 46.

19 P. Sahariah and M. Masson, Biomacromolecules, 2017, 18, 3846.

20 F. Devlieghere, A. Vermeulen and J. Debevere, Food Microbiol., 2004, 21, 703.

21 H. X. Wang, J. Qan and F. Y. Ding, J. Agric. Food Chem., 2018, 66, 395.

22 A. Bernkop-Schnurch and S. Dunnhaupt, Eur. J. Pharm. Biopharm., 2012, 81, 463.

23 L. M. Hu, Y. Sun and Y. Wu, Nanoscale, 2013, 5, 3103.

24 S. M. Wang, L. Ge, X. R. Song, J. H. Yu, S. G. Ge, J. D. Huang and F. Zeng, Biosens. Bioelectron., 2012, 31, 212.

25 W. Suginta, P. Khunkaewla and A. Schulte, Chem. Rev., 2013, 113, 5458.

26 S. S. Voznesenskiy, A. A. Sergeev, A. Y. Mironenko, S. Y. Bratskaya and Y. N. Kulchin, Sens. Actuators, B, 2013, 188, 482 .
27 A. Sergeev and S. Voznesenskiy, Opt. Mater., 2015, 43, 33.

28 R. Signini and S. P. Campana, Polym. Bull., 1999, 42, 159.

29 Comparison Between Peristaltic, Syringe and Pressure Pumps for Microfluidic Applications, https://www.fluigent. com/resources/microfluidic-expertise/advantages-of-pressurebased-microfluidic/system-comparison-for-microfluidic-applications/, (accessed 24/05/2021, 2021).

30 V. Silin, H. Weetall and D. J. Vanderah, J. Colloid Interface Sci., 1997, 185, 94.

31 H. Vaisocherova, E. Brynda and J. Homola, Anal. Bioanal. Chem., 2015, 407, 3927.

32 A. M. Merlot, D. S. Kalinowski and D. R. Richardson, Front. Physiol., 2014, 5, 299.

33 S. C. Chien, C. Y. Chen, C. F. Lin and H. I. Yeh, Biomark. Res., 2017, 5, 31.

34 O. Mori and T. Imae, Colloids Surf., B, 1997, 9, 31.

35 R. Barer and S. Tkaczyk, Nature, 1954, 173, 821.

36 A. Salis, M. Bostrom, L. Medda, F. Cugia, B. Barse, D. F. Parsons, B. W. Ninham and M. Monduzzi, Langmuir, 2011, 27, 11597.

37 S. P. Strand, K. Tommeraas, K. M. Varum and K. Ostgaard, Biomacromolecules, 2001, 2, 1310.

38 M. J. Eddowes, Biosensors, 1987, 3, 1. 